\title{
Perspective
}

PERSPECTIVE Actualité en histoire de l'art

1 | 2006

Antiquité/Moyen Âge

\section{Les palais du pouvoir dans l'Italie médiévale}

\section{Lucio Riccetti}

\section{(2) OpenEdition}

\section{Journals}

Édition électronique

URL : http://journals.openedition.org/perspective/4170

DOI : 10.4000/perspective.4170

ISSN : 2269-7721

Éditeur

Institut national d'histoire de l'art

Édition imprimée

Date de publication : 31 mars 2006

Pagination : 136-140

ISSN : 1777-7852

\section{Référence électronique}

Lucio Riccetti, « Les palais du pouvoir dans l'Italie médiévale », Perspective [En ligne], 1 | 2006, mis en ligne le 31 mars 2018, consulté le 01 octobre 2020. URL : http://journals.openedition.org/perspective/ 4170 ; DOI : https://doi.org/10.4000/perspective.4170 
par les artistes locaux, montrent une certaine originalité dans leur approche plus individuelle et personnelle de la foi ou de la mort. C'est bien seulement lorsque l'œuvre d'art est analysée dans sa globalité, en tenant compte de tous les enjeux de sa commande et de sa réalisation, qu'elle accède véritablement au rang de document historique.

1 On citera, parmi les dernières parutions françaises abordant ce problème : Ludovic Nys, Alain Salamagne, Valenciennes aux $X I V^{e}$ et $X V^{e}$ siècles. Art et histoire, Valenciennes, 1996 ; Catherine Chédeau, Les arts à Dijon au XVI siècle : les débuts de la Renaissance. 1494-1551, Aix-en-Provence, 1999 ; Fragments d'une splendeur. Arras à la fin du Moyen Âge, Annick Notter éd., (cat. expo., Arras, musée des beaux-arts, 2000), Arras, 2000 ; Ludovic Nys, Marc Gil, Saint-Omer gothique : les arts figuratifs à Saint-Omer à la fin du Moyen Âge, 1250-1550, peinture, vitrail, sculpture, arts du livre, Valenciennes, 2004

L'un des ouvrages de référence, toujours utilisé par les historiens de l'art médiéval, est la grande synthèse de Charles Sterling (La peinture médiévale à Paris. 1300-1500, Paris, 1987-1990) que l'auteur, en en relisant les épreuves du deuxième tome avant de mourir, concevait comme la première étape d'un travail plus important sur le royaume de France.

2 Sophie Cassagnes, D'art et d'argent. Les artistes et leurs clients dans l'Europe du Nord (XIV'-XV' siècles), Rennes, 2001.

3 Jonathan Alexander, Paul Binski éd., Age of Chivalry. Art in Plantagenêt England; 1200-1400, (cat. expo., Londres, Royal Academy of arts, 1987-1988), Londres, 1987, et Richard Marks, Paul Williamson éd., Gothic, Art for England. 1400-1547, (cat. expo., Londres, Victoria and Albert Museum, 2003), Londres, 2003

4 Parmi celles-ci, on retiendra l'exposition de 1993 : Frank Günter Zehnder éd., Stefan Lochner, Meister zu Köln : Herkunft Werke - Wirkung, (cat. expo. Cologne, Wallraf-RichartzMuseums, 1993-1994), Cologne, 1993.

5 Il s'agit de lettres et de paiements émis ou reçus par le roi et portant confirmations de contrats, privilèges, dons ou ordres de toutes sortes. De nombreux artistes apparaissent dans ces documents.

6 On regrettera cependant ici - tout en en comprenant les raisons - qu'aient été, par la même occasion, écartés les métiers de la construction et du bâtiment pourtant fort utiles pour comprendre certains phénomènes de polyvalence ou de collaboration artistique par exemple.

7 Trois cent cinquante peintures conservées à Cologne contre 256 à Nuremberg, 133 à Augsbourg, 121 à Ulm. Cette situation s'explique par le désintérêt pour ces œuvres durant la Réforme, qui leur a permis d'échapper au mouvement iconoclaste, et par l'émergence, très tôt, de grandes figures de collectionneurs.

8 La question de la formation locale ou exogène du maître de la Sainte Véronique est l'objet de débats entre Julien Chapuis et Brigitte Corley qu'il n'est pas possible de détailler ici.

Jean-Marie Guillouët, Université de Nantes, jmguillouet@free.fr

\section{Les palais du pouvoir dans l'Italie médiévale}

\section{Lucio Riccetti}

- É. CROuzet-Pavan éd., Pouvoir et édilité. Les grands chantiers dans l'Italie communale et seigneuriale, (Collection de l'École Française de Rome, 302), Rome, 2003. 495 p., ill. n. et b. ISBN : 2-72830633-8; $53 €$.

- P. Boucheron, J. Chiffoleau éd., Les palais dans la ville. Espaces urbains et lieux de la puissance publique dans la Méditerranée médiévale, (Collection d'histoire et d'archéologie médiévales, 13), Lyon, Presses Universitaires de Lyon, 2004. 341 p., 77 ill. n. et b. ISBN : 2-7297-0747-6. $23 €$.

Le débat qui, à partir des définitions d'urbs et de civitas proposées par Isidore de Séville dans les Etymologiae, distinguait deux aspects urbains connexes repris ensuite dans les célèbres catégories établies par Marx ${ }^{1}$ : la "structure de base " (urbs) et la "superstructure" (civitas), mérite d'être dépassé. L'étude des aspects liés à l'urbanisme et à l'architecture des villes médiévales italiennes acquiert, selon la formulation pionnière de Gina Fasoli, une " complexion positive " dans la composition dialectique de cette dichotomie aussi manifeste qu'artificielle. Cette " confusion stimulante et évocatrice ", selon les termes de Fasoli ${ }^{2}$, s'étend de la "nouvelle définition des aspects urbains par leur reconstruction heuristique jusqu'à la représentation cartographique, accompagnée aujourd'hui par les possibilités de la réalité virtuelle, en passant par la ville comme signe iconique de l'imaginaire médiéval " ${ }^{3}$.

Depuis longtemps maintenant, la transformation urbaine et, surtout, le lancement de vastes chantiers ont été reconnus comme champs d'étude privilégiés pour l'étude de la transformation identitaire et celle de la conscience citadine, de même que, ces derniers temps, la formation d'un groupe de personnes qualifiées qui constitua les Fabriques (administration de l'urbanisme) ${ }^{4}$. C'est en effet la participation active à la vie citadine qui à la fois favorise le sentiment d'appartenance à un milieu culturel donné et contribue à le définir : "Sienne me fit", fera dire Dante à Pia de'Tolomei (Le Purgatoire, V, 134).

Les études historiographiques et méthodologiques n'ont pas manqué. Le volume $D^{\prime}$ une ville à l'autre. Structures matérielles et organisation de l'espace dans les villes européennes (XIII ${ }^{e}-X I V^{e}$ siècles), publié en 1989, demeure, aujourd'hui encore, un parfait exemple des nouvelles méthodes d'approche du thème de la ville ${ }^{5}$. En effet, l'impossibilité d'appliquer une grille commune à la diversité des choix 
urbanistiques italiens est mise en évidence, tout comme la nécessité de procéder par étapes successives dans le dessein de faire émerger les particularités des différentes réalités citadines, cette " complexion positive " des choix liés aux nécessités communes : définition d'espaces publics et constructions de bâtiments accueillant les nouveaux pouvoirs.

Depuis cette étude pionnière, la recherche a acquis de nouvelles méthodes qui appréhendent des processus généraux tout en les considérant dans leur contexte particulier, comme le développement des adductions d'eau ${ }^{6}$ et la nécessité de concevoir des édifices à la fois représentatifs et fonctionnels pour les nouvelles magistratures. Dans les décisions communes, il convient d'examiner le rôle prioritaire dévolu aux magistratures itinérantes et considérer la présence d'ouvriers spécialisés, venant souvent d'une autre région, qui est mise à profit dans les grands chantiers urbains. $\mathrm{Si}$, en effet, la carrière de personnalités singulières est souvent connue, plus difficile à cerner est leur milieu culturel : il les conduit pourtant plus ou moins consciemment à endosser un rôle de vecteur des connaissances techniques analogue à celui tenu par les podestats dans la circulation de la culture juridico-administrative et notariale ainsi que dans les commandes de nouveaux lieux de pouvoir ${ }^{7}$. La multiplication des études de cas particuliers est utile pour ne pas isoler les travaux qui, en l'espace de quelques années, prendront corps dans un milieu régional déterminé ; il convient de les considérer comme les témoignages empiriques d'une connaissance généralisée.

Les quatre séminaires organisés à l'École française de Rome sous la direction d'Élisabeth Crouzet-Pavan autour des grands chantiers médiévaux dans l'Italie communale et seigneuriale entre 1994 et 1998 ont abouti à un recueil d'essais qui n'avait pas seulement pour but de vérifier et d'analyser les études sur l'histoire de la cité, mais aussi, et peut-être surtout, comme le souligne la coordinatrice, de faire le point sur le chemin parcouru depuis le volume $D^{\prime}$ 'une ville à l'autre paru en 1989. La nouveauté majeure consiste dans le greffage sur l'histoire de l'urbanisme et de l'architecture, riche d'une longue tradition de thèmes spécifiques liés à la civitas, à l'histoire sociale, à l'horizon culturel et aux aspirations des habitants quant à la définition de l'image même de leur cité. Un peu comme si la ville construite, considérée comme un scénario immuable de révolutions politiques et culturelles, prenait vie et participait à de telles transformations sous l'impulsion de choix, techni- ques autant qu'artistiques, politiques autant qu'administratifs, entrepris par les régimes populaires avec des résultats qui marqueront le tissu social. La construction ou la transformation des villes est liée autant à l'ouverture des chantiers qu'à la définition des appareils législatifs et culturels : très souvent, la définition de la mémoire et de l'image urbaine coïncide. Toutes deux sont liées aux transformations politiques et économiques qui menèrent au pouvoir les régimes populaires. La définition de la cité advient alors à travers la planification urbanistique, la rédaction des libri iurium, ou encore le récit de chroniques citadines.

Le recueil d'études, même s'il est majoritairement associé à l'ouverture et à l'organisation de grands chantiers, tient largement compte de ces aspects à long terme. Ainsi, il met en évidence les changements de direction que la planification des grandes entreprises de construction subit sous l'impulsion des nécessités de la cité et montre comment les stratégies de construction et d'urbanisme sont liées au contrôle du territoire.

Des études strictement urbaines (Maria Rita Silvestrelli sur Pérouse, p. 105-158; C. Caby sur San Michele di Murano, p. 159-193), on passe à celles sur la planification des interventions sur le territoire et sur le contrôle du niveau des eaux à Sienne (Duccio Balestracci, p. 419438), sur la nécessité de prendre en compte la sécurité du contado afin d'éviter les risques de dépeuplement (P. Pirillo, p. 439-459) ou sur des thèmes plus généraux comme la mise en place de stratégies politiques, économiques et techniques, avec l'exemple du Dôme d'Orvieto et la recherche continue de matériaux permettant de mener à bien le chantier (Lucio Riccetti, p. 245-373) ou le réseau de forteresses construites dans le tissu urbain des villes conquises par le cardinal Albornoz (Armand Jamme, p. 375-417). Bien que l'aspect " technique " soit prépondérant, les thématiques mettant en évidence les choix politiques à la base de la définition de la cité ne manquent pas, tel le contrôle des " biens communs " à travers la création de lois spécifiques et de leurs applications (Élisabeth Crouzet-Pavan, p. 11-40). Les modalités et l'apport de fonds publics sont aussi envisagés par rapport à un véritable autofinancement lié à l'exploitation des forêts sur l'Apennin, pour le grand chantier de Sainte-Marie-des-Fleurs (Lorenzo Fabbri, p. 195-244). L'héritage des connaissances techniques et surtout les choix politiques des différentes communes dont les régimes seigneuriaux s'appropriaient ces savoirs en les transformant selon leur propre besoin est étudié 
dans deux cas: Azzone Visconti, et avec lui l'ensemble des régimes seigneuriaux de l'Italie du Nord, interprétera le concept de "biens communs " selon les exigences de la magnificence seigneuriale (Patrick Boucheron, p. 41-77); sur la terre ferme de Vénétie, le rapport entre la ville dominante et le territoire est mis en évidence par l'adoption de modèles architectoniques analogues à ceux de Venise. Ce choix devra être compris soit comme la recherche d'un langage commun conférant un plus grand prestige politique, soit comme un élément possible permettant d'activer les circuits économiques en place pour la construction publique et privée (Donata Degrassi, p. 461-481). Enfin, un dernier thème, central, est également considéré : celui du rapport entre les chantiers réels et leur représentation, permettant de comprendre la propagande politique qui leur est associée (Sandra Baragli, p. 79-104).

Dans la même lignée méthodologique, le volume Les palais dans la ville complète le précédent en traitant un thème spécifique. Les modèles palatiaux n'y sont pas simplement considérés comme un ensemble de formes architectoniques, mais aussi comme des cas d'études pour analyser l'évolution et la diffusion d'une configuration urbaine. L'espace palatial et le contexte urbain d'appartenance sont considérés sur la longue durée dans une aire géographique très vaste : le bassin méditerranéen occidental, du Maroc des villes impériales à l'Italie des communes, en passant par l'Espagne chrétienne et musulmane et la France du Sud. Tout comme dans Pouvoir et édilité, on ne s'arrête pas à l'aspect physique des bâtiments et au rapport qu'ils entretiennent avec la forme de la cité, mais sont considérés également l'usage social des édifices et leur lien avec le pouvoir.

Il s'agit d'un ouvrage très construit qui réunit une série d'apports spécifiques à l'intérieur de quatre champs thématiques envisagés sous un même angle d'approche méthodologique dans laquelle l'analyse archéologique permet à la fois de définir la fonctionnalité de l'édifice et d'en préciser les modèles : une double lecture donc, archéologique et urbanistique d'une part, idéologique et politique d'autre part.

Dans cet ouvrage également, une attention privilégiée a été accordée à la transformation des structures et des fonctions, bien plus qu'à l'analyse topographique. Ce choix met en valeur le rapport dialectique établi entre la cité et le palais. Ainsi dans la première thématique intitulée " héritages et inventions " sont analysées différentes relations dialectiques, comme l'héritage de l'Antiquité au haut Moyen Âge (Andrea Augenti, p. 15-38), ou encore le rôle du palais sur le Palatin dans le développement de la ville de Rome et surtout, la façon dont l'héritage des palais impériaux a été utilisé dans les villes des nouveaux pouvoirs, à Ravenne, Milan, Split et de nouveau à Rome avec la nouvelle définition des édifices et des espaces en rapport avec le Palatin au haut Moyen Âge. Dans le monde islamique, on assiste à l'isolement progressif du pouvoir qui se déplace des palais récents, fondés au centre de la cité (Kairouan) ou dans les environs du forum romain (Cordoue), vers des palais isolés (P. Guichard, p. 39-56). Enfin, nous trouvons, avec les châteaux fortifiés de la vallée du Rhône (Michèle Bois, p. 57-73), un exemple de modèles hybrides qui s'imposera pour représenter le siège du pouvoir épiscopal et sera ensuite exporté en Italie du Sud où il subira encore des modifications qui répondent à la complexité de la réalité urbaine de la ville sicilienne et à ses traditions romaine, byzantine et arabe.

Une large place est accordée aux réalités italiennes, observatoire privilégié permettant d'identifier les modèles, de définir le caractère hybride des styles et des fonctions et de comprendre la topographie des pouvoirs à travers les choix urbanistiques. La seconde partie (Le palais, la ville, le prince) est consacrée à l'Italie. Elle commence par une transition idéale avec le royaume de Sicile (Jean-Marie Martin, p. 77-93), et permet d'affronter l'aspect central de la question de la ville et du palais : le siège du pouvoir épiscopal ${ }^{8}$. Pendant longtemps, il fut l'unique palais de la cité, c'est ce que nous montrent les cas complexes des édifices épiscopaux de Vercelli (Marco Frati, p. 95-108) et de Pise (Gabriella Garzella, p. 109-122) qui restèrent longtemps des obstacles pour la commune qui ne sembla pas avoir la capacité de concevoir un projet d'urbanisme unifié, contrairement à Sienne ou Florence. À Volterra en revanche, le pré de l'évêque devint le cour de la ville (Maria Luisa Ceccarelli Lemut, p. 123-137). À ce sujet, je souhaiterais attirer l'attention sur le cas d'Orvieto : des terrains cédés au chapitre de la cathédrale dans un acte de 1131, en lieu et place de l'actuelle place du Dôme, sont mentionnés sous le nom de Broilo: une zone délimitée par un mur d'enceinte avait donc été utilisée à proximité du siège épiscopal. Cependant, par rapport à la tendance italienne du Nord, où ce même lieu accueillera le palais communal ${ }^{9}$, à Orvieto, on choisira de l'installer dans un lieu diamétralement opposé à celui de l'évêché. En 1131, le Broilo est un terrain qui a changé de 
fonctions; en 1136, on trouve la première attestation de la commune d'Orvieto.

Le troisième champ thématique (Sur les palais des papes)est dédié à ce type d'édifices et sur quatre études, trois concernent le palais des papes d'Avignon (Valérie Theis, Les stratégies d'implantation palatiale dans la région d'Avignon de Jean XXII à Clément VI (1316-1352), p. 165-118; Dominique Carru, Le palais des papes d'Avignon, essai de morphogenèse, p. 189-212 ; Jean-Michel Poisson, Le palais des papes d'Avignon: structures défensives et références symbolique, p. 213-228), caractérisé par une implantation inédite et originale qui sera vite reprise et qui n'est en rien comparable aux choix romains opérés par les souverains pontifes. À Rome, (Pierre-Yves Le Pogam, p. 141-163), l'effort des souverains est dédié à la réorganisation de la ville en fonction des deux palais des papes du Latran et du Vatican situés en périphérie. Une telle requalification advient en accordant non seulement un rôle majeur à la via papalis ou via sacra, qui relie les deux résidences, mais surtout en insérant au cœur même de la ville les fondations religieuses des ordres mendiants et en favorisant l'expansion et la consolidation des familles liées aux papes. Cette politique urbaine mènera à une nouvelle perception de l'espace urbain, transformant Rome en une ville soumise aux souverains pontifes.

Dans la quatrième partie, Le prince, le palais, la représentation, les études se concentrent sur le palais et la façon dont il impose à la ville un sens aigu de la représentation. Venise est un cas emblématique : travers la sédimentation, la durée et la conservation qui maintinrent les différentes strates de l'histoire, on tend à légitimer l'État en conservant des lieux qui résistent à la transformation des institutions, comme si " [à] Venise, se [découvrait] le langage d'une forme d'utopie de la permanence du politique, vers laquelle tendaient, sans y parvenir, les autres cités italiennes " (É. CrouzetPavan, p. 231-248). Les trois palais de Milan, Mantoue et Urbin (P. Boucheron, p. 250-284) sont analysés comme des exemples de la transformation des villes en forme de palais, rappelant les trois Villes idéales d'Urbino, Berlin et Baltimore où la société de cour semble ne vivre qu'à l'intérieur de ces palais, manifestant la nature fondamentalement sacrée du pouvoir. Cette solution n'est pas exclusivement italienne : en effet l'articulation qui s'établit entre la ville et le palais est envisagée dans le même sens pour les villes impériales marocaines (Marianne Barrucand, p. 325-341). De la même façon, le contraste et la contradiction propres à la ville espagnole transforment le palais en une ville dans la ville (Antonio Malpica Cuello, l'Alhambra de Grenade, p. 285-311 ; Rafael Cómez, l'Alcazar de Séville, p. 313-324).

En définitive, après la lecture de ces deux ouvrages, très différents l'un de l'autre, mais qui affrontent la recherche avec des instruments méthodologiques très proches, me revient en mémoire l'affirmation de Wolfgang Braunfels: " Chaque ville nous apprend qui la gouverne et comment elle est gouvernée. " ${ }^{10}$ Le rapport n'est probablement pas si direct, mais la " complexion " des thèmes qu'offrent ces nombreuses contributions est certainement la meilleure voie pour étudier l'histoire des villes du Moyen Âge.

1 Voir Vittorio Franchetti Pardo, Maria Sanfilippo, "Città medievali e storia urbanistica ", dans Quaderni Medievali, 2, 1976, p. 266-281 et p. 266.

2 Gina Fasoli, "Città e storia delle città ", dans Topografia urbana e vita cittadina nell'alto medioevo in Occidente, Spolète, 1973, p. 17-38; republié dans Francesca Bocchi, Antonio Carile, Antonio-Ivan Pini, Scritti di storia medievale, Bologne, 1974, p. 163-179 et p. 20-21 : « confusione ricca di stimoli e di suggestioni ".

3 Alba Maria Orselli, "Le 'laudes civitatum' ", dans Silvia Neri, P. Porta éd., La storia come storia della civiltà, (colloque, Bologne, 1993), Bologne, 1993, p. 81-85; p. 81 : « dalla ridefinizione degli aspetti urbani nella ricostruzione euristica sino alla loro rappresentazione cartografica e ora alla riproposizione nella forme della realtà virtuale, alla città come segno iconico dell'immaginario medievale ".

4 Margaret Haines, Lucio Riccetti, éd., Opera. Carattere e ruolo delle Fabbriche cittadine fino all' inizio dell'età moderna, (colloque, Florence, 1991), Florence, 1996 ; L. Riccetti, " Occasioni di lavoro nel Medioevo : opere pubbliche e Fabbricerie ", dans Metropoli medievali, (colloque, Bologne, 1997), Bologne, 1999, p. 195-205.

5 Jean-Claude Maire Vigueur éd., D'une ville à l'autre. Structures matérielles et organisation de l'espace dans les villes européennes (XIII ${ }^{e}$-XIV siècles), (colloque, Rome, 1986), Rome, 1989.

6 Sur la nécessité des systèmes hydrauliques et la mobilité des techniciens, voir Christer Bruun, Ari Saastamoinen éd., Technology, Ideology, Water: from Frontinus to the Renaissance and Beyond, (colloque, Rome, 2000), Rome, 2003.

7 Au sujet des podestats, nous disposons maintenant du travail monumental suivant : Jean-Claude Maire Vigueur éd., I podestà dell'Italia comunale, Rome, 2000. Une étude équivalente est encore attendue pour la circulation de la maind'œuvre spécialisée. On trouve des indications méthodologiques intéressantes dans l'article de J.-C. Maire Vigueur, « L'essor urbain dans l'Italie médiévale : aspects et modalités de la croissance ", dans Europa en los umbrales de la crisis (1250- 
1350), (colloque, Estella 1994), Pampelune, 1995, p. 171204 ; Carlo Tosco, Architetti e committenti nel romanico lombardo, présenté par Enrico Castelnuovo, Rome, 1997. Pour une étude générale sur le sujet, voir Mathieu Arnoux, Pierre Monnet éd., Le technicien dans la cité en Europe occidentale, 1250-1650, (colloque, Göttingen, 2000), Rome, 2004.

8 Maureen Miller, The Bishop's Palace. Architecture and Autority in Mediaval Italy, Ithaca (NY), 2000.

9 Sur les Broletti, voir P. Racine, "Les Palais publics dans les Communes italiennes (XII ${ }^{\mathrm{e}}$-XIII ${ }^{\mathrm{e}}$ siècles)", dans Le Paysage urbain au Moyen Âge, (colloque, Lyon, 1980), Lyon, 1980, p. 133-153 ; Gigliola Soldi Rondinini, « Evoluzione politicosociale e forme urbanistiche nella Padania dei secoli XII-XIII : i palazzi pubblici ", dans La pace di Costanza 1183. Un difficile equilibrio di poteri fra società italiana ed Impero, (colloque, Milan/ Piacenza, 1983), Bologne, 1984, p. 85-98 ; Carlo Ghisalberti, "Il Broletto nel quadro dello sviluppo urbano della Milano comunale ", dans Arte lombarda, III, 1989, p. 73-82 ; Giancarlo Andenna, « La simbologia del potere nelle città comunali lombarde : i palazzi pubblici ", dans Paolo Cammarosano éd., Le forme della propaganda politica nel Due enel Trecento, (colloque, Trieste, 1993), Rome, 1994, p. 369-393.

10 Wolfgang Braunfels, Urban Design in Western Europe. Regime and Architecture, 900-1900, Chicago/Londres, 1976 (1988), p. 2 : "Each city lets us know who governs it and how it is governed".

Lucio Riccetti, Université de Pérouse, ricetti@unipg.it 